\title{
Intraarticular Injection of Infliximab-Loaded Thermosensitive Hydrogel Alleviates Pain and Protects Cartilage in Rheumatoid Arthritis
}

This article was published in the following Dove Press journal: Journal of Pain Research

\author{
Weiying Chen ${ }^{1,2}$ \\ Zuhao $\mathrm{Li}^{2,3}$ \\ Zhenhong Wang ${ }^{2}$ \\ Hong Gao' \\ Junyun Ding ${ }^{2}$ \\ Zhenzhou $\mathrm{He}^{1,2}$ \\ 'School of Anesthesiology, Third \\ Affiliated Hospital of Guizhou Medical \\ University, Guiyang 550004, People's \\ Republic of China; ${ }^{2}$ Department of \\ Anesthesiology, South Campus, Renji \\ Hospital, School of Medicine, Shanghai \\ Jiao Tong University, Shanghai 20III2, \\ People's Republic of China; ${ }^{3}$ Orthopaedic \\ Medical Center, The Second Hospital of \\ jilin University, Changchun I3004I, \\ People's Republic of China
}

Purpose: Pain and cartilage destruction caused by rheumatoid arthritis (RA) are major challenges during clinical treatment. Traditional systemic administration not only has obvious side effects but also provides limited relief for local symptoms in major joints. Local delivery of therapeutics for RA treatment is a potential strategy but is limited by rapid intraarticular release.

Materials and Methods: In this study, we prepared a thermoresponsive injectable hydrogel by mixing pluronic F127 (F127) and hyaluronic acid (HA) with poly ( $\gamma$-glutamic acid) (PGA) incorporating infliximab (IFX), a new generation monoclonal antibody drug. We investigated the biocompatibility of the hydrogel and its IFX release profile. In vivo, we studied the clinical manifestations (articular skin temperature and joint diameter), detected cytokines in the synovial fluid and cartilage, performed behavioral studies on pain relief, and evaluated the cartilage protection effect.

Results: A thermoresponsive hydrogel was successfully prepared by mixing F127, HA, and PGA with injectable properties. The F127-HA-PGA hydrogel had a porous structure with interconnected pores. The infliximab-loaded thermosensitive hydrogel exhibited good biocompatibility and biodegradability and sustained release properties. Intraarticular injection of the IFX-loaded F127-HA-PGA hydrogel could alleviate the expression of inflammatory cytokines, such as tumor necrosis factor- $\alpha$ (TNF- $\alpha$ ), interleukin-1 $\beta$ (IL-1 $\beta)$, interleukin-6 (IL-6), and interleukin-17 (IL-17), in the synovial fluid and cartilage as well as relieve pain and inhibit cartilage destruction in RA.

Conclusion: The double effect on pain relief and cartilage protection indicated the significant potential of the IFX-loaded injectable hydrogel for RA treatment in major joint lesions.

Keywords: rheumatoid arthritis, infliximab, hydrogel, intraarticular injection, control release

\section{Introduction}

Rheumatoid arthritis (RA), which affects about $1-2 \%$ of the global adult population, is a chronic arthritic disease characterized by synovial proliferation, joint swelling and pain, and cartilage destruction, eventually leading to joint stiffness, deformity, and dysfunction. ${ }^{1,2}$ In addition to restricted mobility and disability caused by the persistent destruction of articular cartilage due to chronic inflammation and uncontrollable synovial hyperplasia, pain is another factor plaguing patients. ${ }^{3}$ In the microenvironment of arthritis, local immune cells are known to secrete inflammatory cytokines, such as tumor necrosis factor- $\alpha$ (TNF- $\alpha$ ),
School of Anesthesiology, Third Affiliated Hospital of Guizhou Medical University, Guiyang 550025, People's Republic of China

Email2169617@qq.com

Zhenzhou He

Department of Anesthesiology, South

Campus, Renji Hospital, School of

Medicine, Shanghai Jiao Tong University,

Shanghai 201 100, People's Republic of

China

Email sandyhezz@I26.com
Journal of Pain Research 2020:13 3315-3329

3315

DovePress if in $>$

(c) (i) (5) 2020 Chen et al. This work is published and licensed by Dove Medical Press Limited. The full terms of this license are available at https://www.dovepress.com/terms.php (c) 1 BY NC and incorporate the Creative Commons Attribution - Non Commercial (unported, v3.0) License (http:///creativecommons.org/licenses/by-nc/3.0/). By accessing the work you hereby accept the Terms. Non-commercial uses of the work are permitted without any further permission from Dove Medical Press Limited, provided the work is properly attributed. For permission for commercial use of this work, please see paragraphs 4.2 and 5 of our Terms (https://www.dovepress.com/terms.php). 
interleukin-1 $\beta$ (IL-1 $\beta$ ), interleukin-6 (IL-6), and interleukin-17 (IL-17), thus directly sensitizing nociceptors, which have the potential to cause severe pain. ${ }^{4,5}$

The therapeutic objectives of RA are to suppress synovial inflammation, alleviate joint pain and swelling, prevent damage to joint and articular substructures, halt disease progression, and reinstate joint mobility and function. ${ }^{6}$ Traditional therapy strategies for RA include administration of nonsteroidal anti-inflammatory drugs (NSAIDs), corticosteroids, and disease-modifying antirheumatic drugs, which have been reported to play a positive role in the treatment of RA but have also been associated with a series of unbearable adverse effects. ${ }^{7}$ New generation monoclonal antibody drugs, such as antitumor necrosis factor- $\alpha$ antibodies (anti-TNF $\alpha$ Abs) constitute a significant progress in the clinical treatment of RA. However, long-term administration might lead to ontarget toxicities, such as severe infections (tuberculosis or septic arthritis) and malignancy. ${ }^{8}$

Intraarticular administration is an important supplement of traditional therapy to control RA symptoms in large joints, and this has been shown to decrease drugrelated systemic side effects and might be especially applicable in delivering drugs with low bioavailability. ${ }^{9}$ However, rapid clearance of local drugs means that multiple injections are required to achieve the desired therapeutic effect. Accordingly, this repeated administration leads to poor patient compliance and more complications. ${ }^{10,11} \mathrm{In}$ this case, encapsulating drugs in the delivery system for intraarticular sustained release has been suggested as the ideal strategy. ${ }^{12,13}$ In particular, thermoresponsive hydrogels consisting of Pluronic F127 and hyaluronic acid (HA) were demonstrated to be suitable for intraarticular injection for arthritis owing to their encapsulation efficiency and controlled and stable release profiles. ${ }^{14-16}$ The F127HA hydrogel could be used for sustained-release drugs; however, its stability is not clear, with the usual release time of only approximately 3 days. However, by introducing poly ( $\gamma$-glutamic acid) (PGA) into the F127-HA mixture, the stability of the F127-HA hydrogel could be increased, especially through regulation of the molecular weight and concentration of PGA. Therefore, the addition of PGA was shown to significantly enhance the stability of these hydrogels, thus providing the possibility for a more durable release. ${ }^{17}$

In the present study, we prepared a thermoresponsive injectable hydrogel by mixing pluronic F127 and HA with PGA and investigated the cartilage protection and effects of local delivery of infliximab (IFX) in a RA rabbit model. Pluronic-based hydrogels are good candidates for injectable hydrogels owing to their thermosensitive sol-gel transition behavior. They are soluble at lower temperatures $\left(\mathrm{eg}, 4-10^{\circ} \mathrm{C}\right)$ and therefore have injectable properties. Once they reach a higher temperature (eg, entering the human body, $37^{\circ} \mathrm{C}$ ), they have been shown to quickly form gels, thus ensuring excellent retention in the joint. ${ }^{18}$ In particular, HA, a linear polysaccharide composed of D-glucuronic acid and $\mathrm{N}$-acetylglucosamine, is an attractive natural polymer material owing to its high biocompatibility and controlled degradability. ${ }^{19}$ Moreover, HA is also a key component of articular cartilage and can lubricate the joint after its intraarticular injection. Furthermore, note that HA intraarticular injection could significantly moderate the inflammatory response of synovial tissue, promote the healing and regeneration of articular cartilage, and alleviate pain, thus contributing to the recovery of disease-affected tissue. ${ }^{20}$ As a model drug for encapsulation, IFX, targeting TNF- $\alpha$ in the disease region, could significantly alleviate pathological inflammation and efficiently inhibit RA progression. ${ }^{21}$ Therefore, we predicted that intraarticular injection of an IFX-loaded F127-HAPGA hydrogel could alleviate local inflammation of joints, relieve pain, and reduce cartilage destruction in RA.

\section{Materials and Methods Ethical Approval}

All animal procedures were performed in accordance with the guidelines for the Care and Use of Laboratory Animal Experience of Shanghai Jiaotong University and were approved by the Animal Ethics Committee of Renji Hospital Affiliated to Shanghai Jiaotong University.

\section{Materials}

Pluronic F127 (F127, Mw 12.6 kDa), HA (Mw 2100 $\mathrm{kDa})$, poly ( $\gamma$-glutamic acid) (PGA, Mw $2000 \mathrm{kDa}$ ), ovalbumin (OVA, $\approx 99 \%$ ), Freund's adjuvant, and all ELISA kits were obtained from Sigma-Aldrich (St. Louis, Missouri, USA). Dulbecco's modified Eagle medium (DMEM, low glucose), fetal bovine serum, and streptomycin-penicillin were supplied by Gibco Life Technologies (Grand Island, New York, USA). Rabbit chondrocytes (CP-Rb002) were obtained from Procell Life Science \& Technology Co., Ltd. (Wuhan, China). Cell Counting Kit (CCK-8) and Calcein-AM/PI were purchased from Biobetimes Biotechnology Co., Ltd. 
(Changsha, China). Paraformaldehyde solution (4\%) was obtained from Xilong Chemical Co., Ltd (Yulin, China). Hematoxylin, eosin, and toluidine blue were obtained from Thermo Fisher Scientific Co., Ltd (Shanghai, China). Triton X-100 was supplied by Solarbio Science \& Technology Co., Ltd. (Beijing, China). Antibodies used for immunofluorescence were purchased from Abcam (Cambridge, UK).

\section{Hydrogel Preparation and Drug Encapsulation}

To prepare thermoresponsive injectable hydrogels, F127 (17 wt $\%)$ and HA (1 wt $\%$, Mw $2100 \mathrm{kDa})$ were dissolved in deionized water with PGA (10 wt $\%$, Mw $2000 \mathrm{kDa})$ at $4^{\circ} \mathrm{C}$ for 1 day using a rotary shaker (Barnstead International, Dubuque, IA, USA). When ingredients were fully dissolved, infliximab $(0.1 \mathrm{wt} \%)$ was added and well mixed using a rotary shaker.

\section{Characterization}

Rheological measurement of the F127-HA-PGA hydrogel was performed using a rheometer (Malvern, UK) with temperature increase at a frequency of $1 \mathrm{~Hz}$. During the test, the temperature was set from $10^{\circ} \mathrm{C}$ to $45^{\circ} \mathrm{C}$, with the heating rate being $1^{\circ} \mathrm{C} / \mathrm{min}$. The morphology of the injectable hydrogel was observed using a scanning electron microscope (SEM) (JEOL JSM-6700F, Japan) operated at an acceleration voltage of $3 \mathrm{kV}$. In brief, hydrogels with or without IFX were frozen in liquid nitrogen for $5 \mathrm{~min}$, followed by freeze-drying; the samples were cut to size and the superficial part was removed and then coated with a conductive layer of sputtered gold for SEM observation. In vivo degradation was performed by subcutaneous injection of the hydrogel in rats. Briefly, $1 \mathrm{~mL}$ hydrogel was subcutaneously injected into the backs of Sprague-Dawley (SD) rats. Then, rats were sacrificed at predetermined time points. Looking for the undegraded part of the hydrogel under the skin, we carefully dissected it with ophthalmic scissors and forceps. Then, the surrounding fat and fascia were cleaned, and the intact undegradable hydrogel was weighed. The weight of the hydrogel blocks obtained immediately after injection was recorded as $100 \%$. Then, skin was collected and fixed in $4 \%(\mathrm{w} / \mathrm{v})$ paraformaldehyde solution. Hematoxylin and eosin (H\&E) staining was performed for in vivo biocompatibility analysis. All optical images were recorded using a digital camera (Canon EOS 550D, Japan).

\section{Release Profiles of Infliximab from Hydrogel}

To detect the drug release rate, IFX-loaded hydrogels were prepared and soaked in $5 \mathrm{~mL}$ phosphate-buffered saline (PBS, $0.01 \mathrm{M}, \mathrm{pH}$ 7.0). Preparations were shaken at $50 \mathrm{rpm}$ and $37^{\circ} \mathrm{C}$ in sterile glass bottles. At the predetermined time point, all supernatants were removed and stored at $-20^{\circ} \mathrm{C}$ for detection, and bottles were replenished with equivalent volume of PBS for subsequent drug release studies.

The concentration of released IFX in PBS was analyzed using an ELISA kit and a microplate reader (Multiskan EX, Thermo Fisher Scientific Inc., Shanghai, China) at $490 \mathrm{~nm}$. The IFX release profiles were calculated in a cumulative manner with the following equation: ${ }^{22}$

Cumulative IFX released $(\%)=$ cumulative released amount of IFX/initial amount of IFX $\times 100 \%$

\section{Biocompatibility}

To evaluate the cell biocompatibility of the hydrogel and the IFX-loaded hydrogel, the proliferation of chondrocytes was analyzed using a CCK-8 assay. Briefly, chondrocytes were seeded in 48-well plates at a density of $5 \times 10^{4}$ cells/well and incubated at $37^{\circ} \mathrm{C}$ in a $5 \% \mathrm{CO}_{2}$ atmosphere. One day after seeding, cells were completely attached, and the growth medium was discarded and replaced with sample extracts (hydrogel and IFX-loaded hydrogel were soaked in fresh DMEM for $3 \mathrm{~d}$ and the respective extracts were collected in advance). No hydrogel extracts were used in the control group. After incubation for 1, 4, and 7 days, CCK-8 solution was added to the samples, and the samples were incubated at $37^{\circ} \mathrm{C}$ for $2 \mathrm{~h}$. Finally, $100 \mu \mathrm{L}$ of each specimen solution was transferred into a 96-well plate and measured at $450 \mathrm{~nm}$ using a microplate reader. To evaluate cell viability in the IFXloaded hydrogel, live/dead assay was performed 4 days after cell seeding. Samples were incubated with $1 \mathrm{mM}$ calcein$\mathrm{AM}$ for $1 \mathrm{~h}$ and then incubated with $1 \mu \mathrm{g} / \mathrm{mL}$ propidium iodide (PI) for $5 \mathrm{~min}$ at $37^{\circ} \mathrm{C}$. Next, images of cell staining were taken using a fluorescence microscope (Olympus IX71, Tokyo, Japan).

In vivo biocompatibility analysis was performed as previously described (Characterization section). H\&E staining of the skin was performed at different time points after subcutaneous injection of the hydrogel.

\section{Induction of RA}

New Zealand white rabbits (male, 2.5-3.0 kg, 5-mo-old, $\mathrm{n}=30$ ) were prepared as an RA model for in vivo 
experiments. Ovalbumin (OVA) solution $(20 \mathrm{~g} / \mathrm{L}, 1 \mathrm{~mL})$ was prepared with PBS and then mixed with an equal volume of Freund's complete adjuvant (CFA) to be used to generate the RA model. Briefly, the mixed solution was emulsified at $4^{\circ} \mathrm{C}$ and subcutaneously injected into the dorsal region of rabbits weekly for three weeks at 5 points under sterile conditions. On the fourth week, the antigeninduced arthritis model was prepared by injecting $0.5 \mathrm{~mL}$ mixed OVA/CFA emulsion into the left knee joint cavity of rabbits.

Rabbits were randomly divided into three groups. After successful generation of the RA model (1 week after OVA/ CFA intraarticular injection), the left knee joints of RA rabbits underwent three kinds of therapies - intraarticular injection with $0.5 \mathrm{~mL}$ physiological saline solution as control (NC group), $0.5 \mathrm{~mL}$ hydrogel (HG group), and $0.5 \mathrm{~mL}$ IFX-loaded hydrogel (HG-IFX group). These RA rabbits were sacrificed for the following studies 6 weeks after intraarticular injection therapies.

\section{Measurement of Clinical Manifestations}

To investigate the classical symptoms of the RA model animals during the in vivo experiment, the articular skin temperature and joint diameter of the left knees were recorded using an electronic thermometer (Yuwell THP79JU, Shanghai, China) and micrometer caliper (Links, Harbin, China). All detections were performed biweekly at a static state and $25^{\circ} \mathrm{C}$.

\section{Detection of Cytokines in Synovial Fluid}

Two and six weeks after intraarticular injection of therapeutic agents, the synovial fluid was extracted from the articular cavity of rabbits using syringes. The concentrations of TNF- $\alpha$, IL-1 $\beta$, IL- 6 , and IL-17 in the synovial fluid were measured using ELISA kits following the manufacturer's instructions.

\section{Behavioral Studies of Pain Relief}

To study the pain relief elicited by the intraarticular injections, weight-bearing capacity (WBC) and paw withdrawal threshold (PWT) tests were carried out to detect the gait and pain threshold. $^{23}$ A 3D gait analysis system (Kinama Tracer, Japan) was used to quantify the WBC values of the hind limbs of rabbits while freely walking. Briefly, RA rabbits walked along a runway equipped with mechanical sensors. The sensors can record the ground reaction force for each foot, reacting to the weight supported by the corresponding limb. According to the ground reaction force, the weight- bearing index (WBI) was calculated using the following formula:

WBI $=$ ipsilateral weight/(ipsilateral weight + contralateral weight) $\times 100 \%$

In addition to study the mechanical allodynia (hypersensitivity), PWT was detected using a von Frey filament (Ugo Basile, Varese, Italy) as described in a previous study. ${ }^{23}$ Briefly, the withdrawal threshold was gauged by the force exerted by the von Frey filament ranging from 0 to $40 \mathrm{~g}$ with a $0.2 \mathrm{~g}$ accuracy. An acicular stimulation was delivered to the mid planta pedis of the left hind limbs through the mesh at the bottom of the cage, with the withdrawal threshold being automatically recorded. The paw sensitivity threshold was regarded as the minimum force leading to a strong and immediate withdrawal reflex of the paw. Motion-related random movements were not regarded as withdrawal reactions. Stimulation was applied on each left posterior paw every $5 \mathrm{~s}$. These tests were repeated 5 times, and the final results were obtained by calculating the average values.

\section{Histological and Immunohistochemical Evaluation}

RA rabbits were anesthetized and sacrificed by intracardiac overdose of sodium pentanal six weeks after intraarticular injection therapies. The left distal femoral joints and synovium tissues were carefully collected and fixed in $4 \%$ paraformaldehyde for further tests.

Synovial tissues were embedded in paraffin for sectioning, and $\mathrm{H} \& \mathrm{E}$ staining was performed to evaluate the inflammatory response. Bone tissue samples of the knee joint were decalcified with $0.5 \mathrm{M}$ EDTA solution for 1 month and embedded in paraffin to obtain slices with a thickness of $5 \mu \mathrm{m}$. According to standard protocols, samples were stained with H\&E and toluidine blue. Immunohistochemical staining of collagen type I (COL I) and collagen type II (COL II) was performed to detect the composition of cartilage collagen. Further, immunofluorescence staining was performed to detect the expression of TNF- $\alpha$, IL-1 $\beta$, IL-6, and IL-17 in the cartilage.

\section{Statistical Analyses}

All results were expressed as mean \pm standard deviation from at least three independent experiments. Results were analyzed via either the Student's $t$-test or one-way analysis of variance using the statistical software SPSS 19.0 (SPSS Inc., Chicago, 
IL, USA). A $p<0.05$ was considered statistically significant between groups. All histological images were analyzed using Image Pro Plus 6.0 (NIH, Bethesda, MD, USA).

\section{Results and Discussion}

\section{Hydrogel Preparation and Drug Encapsulation}

The thermoresponsive injectable hydrogel can be easily formed by mixing F127, HA, and PGA in deionized water at $4^{\circ} \mathrm{C}$. The sol-to-gel phase transformation process is shown in Figure 1A. The hydrogel was soluble at lowtemperatures $\left(4^{\circ} \mathrm{C}\right)$, being injectable under these conditions. However, under body temperature $\left(37^{\circ} \mathrm{C}\right)$ conditions, the hydrogel was observed to quickly form a gel state, thus ensuring excellent retention in the joint. The polymer hydrogel solution could be ejected through a 22 -gauge needle (internal diameter $\approx 410 \mu \mathrm{m}$ ) without clogging at $4^{\circ} \mathrm{C}$ and was shown to form a stable gel state within a few seconds at $37^{\circ} \mathrm{C}$ (Figure 1B). This viscoelastic property of the hydrogel was observed in the temperature range from $10^{\circ} \mathrm{C}$ to $45^{\circ} \mathrm{C}$. Figure $1 \mathrm{C}$ indicates that both the storage modulus $\left(G^{\prime}\right)$ and loss modulus $\left(G^{\prime \prime}\right)$ of the hydrogel rapidly increased after $26^{\circ} \mathrm{C}$, suggesting that the temperature of the solution to complete the solgel transition was $26^{\circ} \mathrm{C}$. Drug administration by intraarticular injection is an emerging popular treatment for arthritis. This strategy of drug delivery minimizes systemically effects and maximizes local effects. The injectability of this formulation is safe and with minimal damage to tissues; moreover, it is convenient for repeated administration. In addition, we observed the morphology of the hydrogel using SEM (Figure 1D). Accordingly, we found that the F127-HA-PGA hydrogel exhibited a porous structure, with interconnected inner pores. Moreover, the pore structure of the hydrogel maintained its original state even after loading of IFX (Figure 1E). We performed in vivo degradation experiments on SD rats. As shown in Figure 1F, it took approximately 24-30 days from subcutaneous injection of the hydrogel to its complete degradation.

\section{Infliximab Release}

The drug release profile is considered to be one of the most important characteristics in accessing the biological efficacy of drug delivery systems. We accordingly observed the release behavior of loaded-IFX from the hydrogel over a period of $28 \mathrm{~d}$ (Figure 2). On the first day, the percentage of released IFX was shown to be $25.2 \% \pm 1.9 \%$, with the release rate gradually decreasing in the following days, exhibiting a sustained and controlled drug-release curve. On the 14th day, the total amount of released IFX was increased to $63.3 \% \pm 2.6 \%$, after which the release profile did not significantly increase. Ultimately, $66.1 \% \pm 2.0 \%$ was demonstrated to be the cumulative released amount of IFX on the 28th day.

Although F127-HA hydrogel can be used for sustained-release drugs, its stability is known to be poor, with a release time of usually only approximately 3 days. However, by introducing PGA into the F127-HA mixture, the stability of the F127-HA hydrogel could be increased, especially through regulation of the molecular weight and concentration of PGA. Herein, a large molecular weight $(2000 \mathrm{kDa})$ and high concentration $(10 \mathrm{wt} \%)$ PGA was incorporated into the F127-HA solution, thus providing a durable release profile. ${ }^{17}$ Our hydrogel could remain subcutaneously stable for 24-30 days after which it would be completely degraded. The release of IFX was demonstrated to mainly occur through drug diffusion and hydrogel degradation. ${ }^{24}$ Drug diffusion might play a major role in the initial release of the drug, because the hydrogel has not been significantly degraded at this stage. In addition, the slight burst release observed during the initial stage might be related to the drug adsorbed on the composite scaffold surface by electrostatic interaction, whereas in the subsequent release period, the degradation of the hydrogel might become the main factor promoting release. The slow gel degradation was shown to enable the continuous and controllable release of IFX. ${ }^{25}$ Our results indicated that the IFX-loaded hydrogel could release IFX continuously within approximately 28 days, thus making it an excellent local drug sustained delivery system for RA.

\section{Biocompatibility of Infliximab-Loaded Hydrogel}

The biocompatibility of the hydrogel with or without IFX was tested using a CCK- 8 assay. Our results indicated that the proliferative capability of chondrocytes treated with extracts from hydrogel or IFX-loaded hydrogel was equal to that of the control group (Figure 3A). Furthermore, when we performed a live/dead assay we found that all groups exhibited good cell viability (Figure 3B). In addition, we tested the in vivo biocompatibility of the hydrogel using $\mathrm{H} \& \mathrm{E}$ staining. 

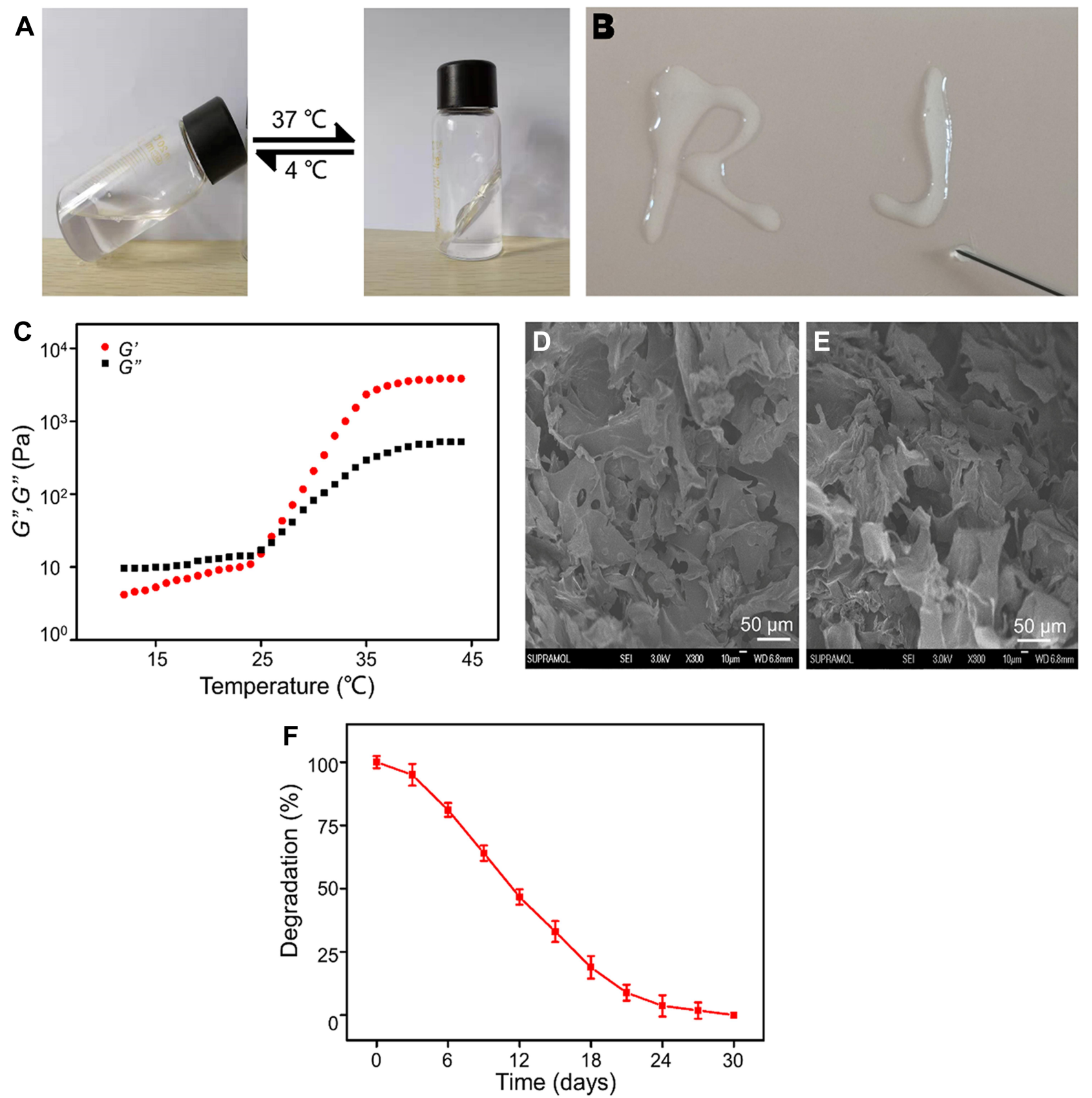

Figure I Characterization of hydrogel. (A) The optical images of the gelation progress. (B) Photo of ejecting hydrogel through a $22 \mathrm{G}$ syringe needle from syringe without clogging. (C) Storage modulus $\left(G^{\prime}\right)$ and loss modulus $\left(G^{\prime \prime}\right)$ of hydrogel as a function of temperature. (D) Morphologies of hydrogel. (E) Morphologies of IFX-loaded hydrogel. (F) Quantitative statistics of undegraded hydrogel weight.

Accordingly, we observed the occurrence of very slight inflammation $20 \mathrm{~min}$ after subcutaneous injection (recorded as $0 \mathrm{~d}$ ), based on the infiltration of a small amount of lymphocytic and neutrophil cells. This inflammation was shown to be gradually alleviated with hydrogel degradation. After 18 days, we could not detect any obvious infiltration of inflammatory cells at the injection site or surrounding tissue (Figure $3 \mathrm{C}$ ). These results suggested that the drug delivery system exhibited good biocompatibility and non-cytotoxicity both in vitro and in vivo.

\section{Clinical Manifestations}

To investigate the therapeutic effect of IFX-loaded hydrogel on RA, we administered $0.5 \mathrm{~mL}$ saline, hydrogel, or IFX-loaded hydrogel into the left knee joints of rabbits $7 \mathrm{~d}$ after OVA-CFA intraarticular injection. 


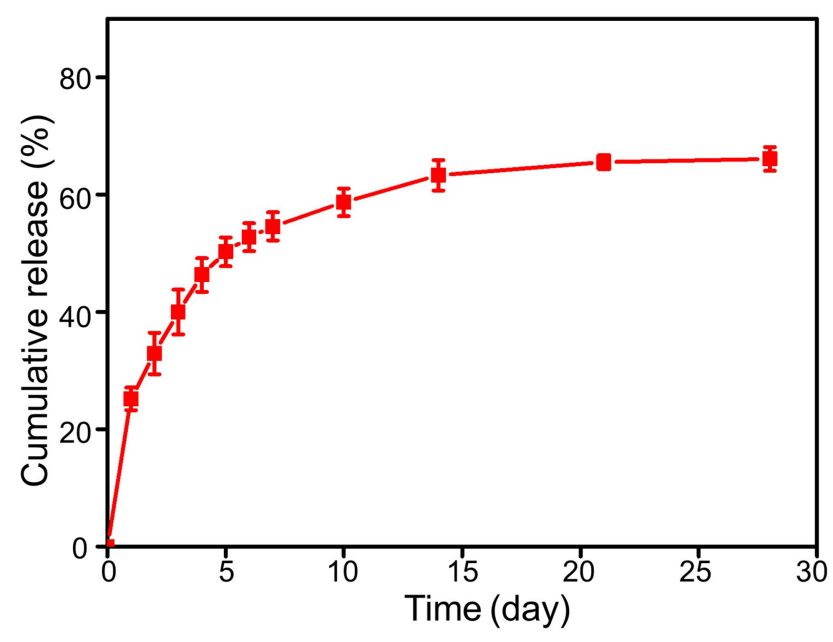

Figure 2 Cumulatively released IFX amount at each time point from hydrogel for 28 days.

In patients with RA, articular surface temperature and joint swelling are important clinical symptoms caused by local inflammatory reactions and synovial hyperplasia. ${ }^{26}$ In our study, the articular surface temperature and joint swelling of all rabbits in the first four weeks (before injecting the IFX therapeutic agent) were found to be elevated after injection of the OVA/CFA emulsion, without any statistically significant differences being observed among the NC, HG, and HG-IFX groups. However, after injecting the IFX-loaded hydrogel, the articular surface temperature and swelling degree were demonstrated to be significantly decreased $(\mathrm{p}<0.05)$ compared with $\mathrm{NC}$ and $\mathrm{HG}$ at 2, 4, and 6 weeks (Figure 4A and B). However, as verified by the following gross findings and histopathological analysis, both their surface temperature and joint diameter were observed to be increased even before OVACFA injection, suggesting that intraarticular delivery of IFX could alleviate but not completely remove local inflammation and synovial hyperplasia. In addition, the HG group was shown to exhibit a tendency to reduce surface temperature (at 4 week, $\mathrm{p}<0.05$ ) and joint swelling (at 6 week, $\mathrm{p}<0.05$ ) compared with the $\mathrm{NC}$ group.

HA-based hydrogels could also have therapeutic potential in RA because HA as a viscosupplement is known to enhance the viscosity and elastic nature of the synovial fluid, thus working as a lubricant. ${ }^{27}$ In addition, HA has
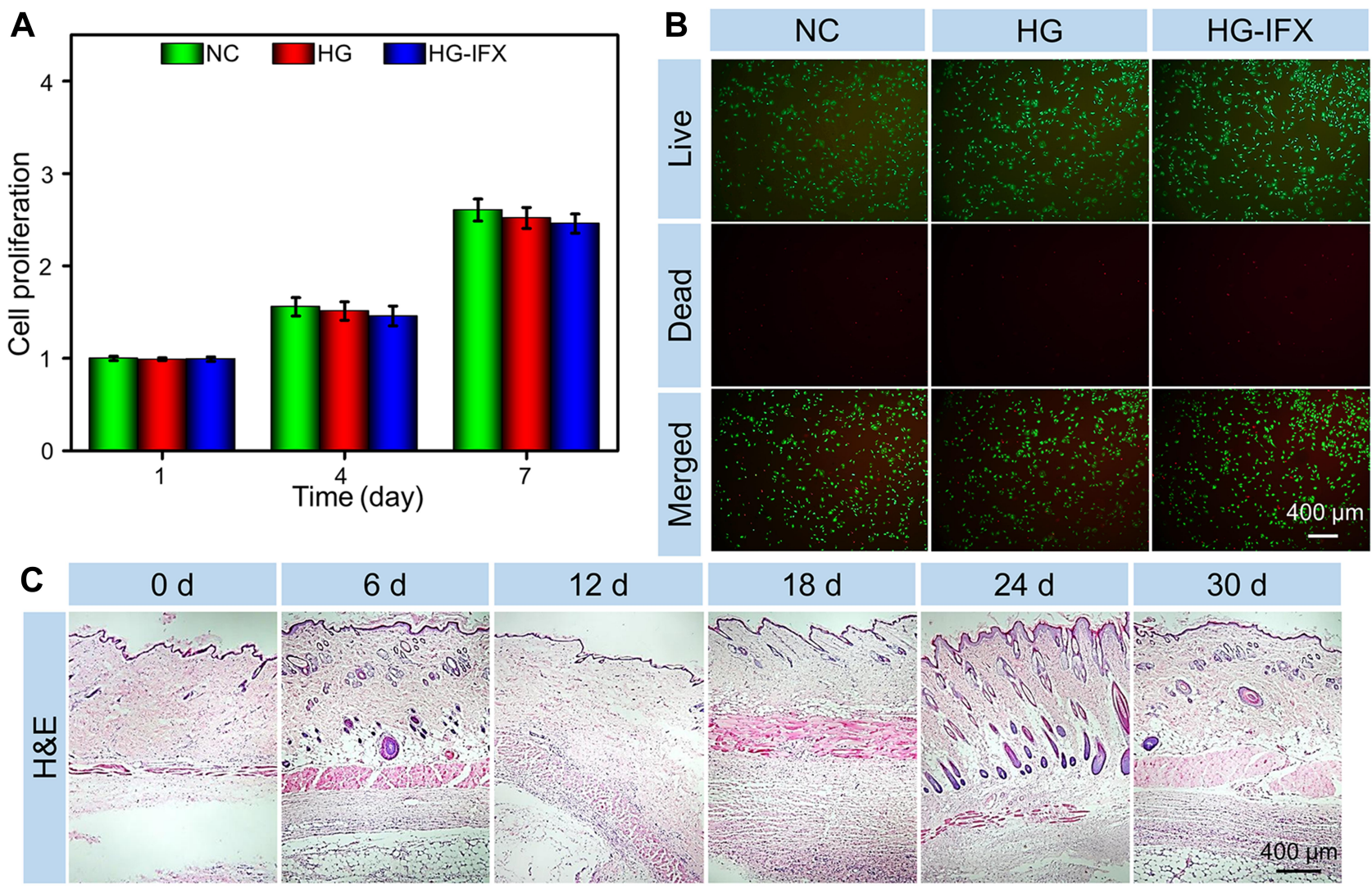

Figure 3 IFX-loaded hydrogel was biocompatible with chondrocytes. (A) The cell proliferation assay was studied with chondrocytes for I, 4 and 7 days by CCK-8 assay. (B) Representative fluorescence microscopy images after live/dead staining of chondrocytes incubated for 4 day. (C) H\&E staining showed no obvious inflammatory reaction in the surrounding muscles and subcutaneous tissues after subcutaneous injection of hydrogel. 

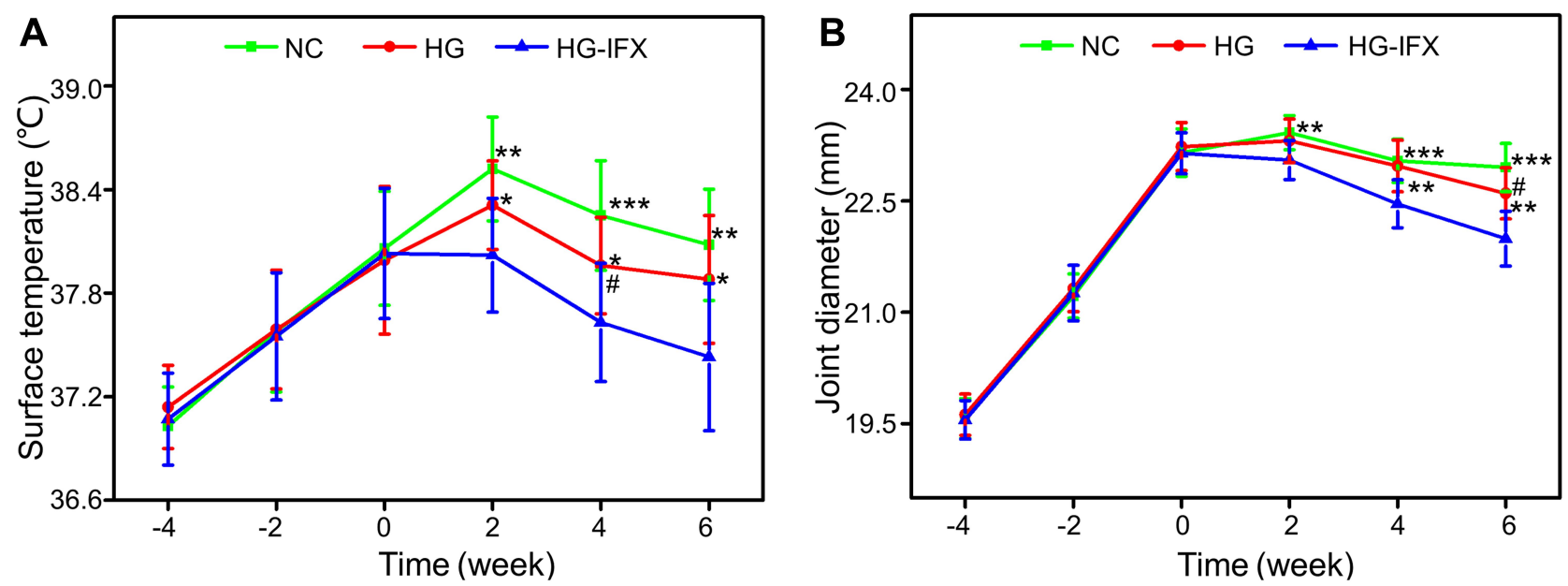

Figure 4 Measurements of $(\mathbf{A})$ surface temperature and $(\mathbf{B})$ joint diameter of left knee after induction of RA and injection of IFX-loaded hydrogel $\left({ }^{*} \mathrm{p}<0.05\right.$, $* * \mathrm{p}<0.0 \mathrm{I}$, $*_{* *} \mathrm{p}<0.00$ I compared with HG-IFX, ${ }_{\mathrm{p}}^{*}<0.05$ compared with NC).

also been reported to inhibit the secretion of enzymes, free radicals, and inflammatory mediators in arthritis as well as hinder the destruction of articular cartilage induced by these substances and accelerate the synthesis and repair of articular cartilage. ${ }^{19,28-30}$ Based on these findings, it could be assumed that intraarticular injection therapy using an IFX-incorporated HA-based hydrogel could significantly alleviate clinical symptoms.

\section{Infliximab-Loaded Hydrogel Alleviated \\ Pain}

We recorded the WBI values two and six weeks after intraarticular injection of $0.5 \mathrm{~mL}$ of therapeutic agents into the knees of RA rabbits. Quantitatively, the WBI values in the $\mathrm{NC}, \mathrm{HG}$, and HG-IFX groups were observed to be $35.2 \% \pm 2.0 \%, 38.2 \% \pm 2.0 \%$, and $40.2 \% \pm 2.9 \%$ at two weeks and $33.5 \% \pm 3.1 \%, 36.0 \% \pm 2.0 \%$, and $39.3 \%$ $\pm 2.4 \%$ at six weeks, respectively. A WBI value less than $50 \%$ indicated reduced weight bearing of the treated leg and increased contribution of the contralateral leg in bearing weight. We considered that the WBI value was reduced in the limb of the inflamed joint because weight bearing aggravated the sensation of pain, a major symptom of arthritis. Intraarticular injection of HA alone into the joints is known to relieve pain by attenuating the inflammatory response through the direct action of HA or by reinforcing the viscosity of the joint fluid. ${ }^{31}$ Therefore, it was reasonable that the WBI values of the HG and HGIFX groups were shown to be significantly higher than those of the NC group at two weeks $(\mathrm{p}<0.05)$.
Moreover, the WBC of the HG-IFX group showed a better result at six weeks post-injection compared with that of the $\mathrm{NC}$ and $\mathrm{HG}$ groups (Figure 5A). In addition, we observed that the ipsilateral PWT of NC-treated rabbits decreased to $7.6 \pm 1.8 \mathrm{~g}$ at week 2 and to $7.2 \pm 1.8 \mathrm{~g}$ at week 6 , both of which were significantly lower compared with those of the HG-IFX-treated group (10.6 $\pm 1.8 \mathrm{~g}$ at week 2 and $11.8 \pm 2.7 \mathrm{~g}$ at week 6 ). With the assistance of IFX, the HG-IFX group was also demonstrated to significantly reduce the CFA-induced hypersensitivity at six weeks compared with the HG group (Figure 5B).

The mechanisms of peripheral pain are known to include the direct activation of nociceptors, as well as the sensitization of nociceptors by inflammation in the articular cavity. ${ }^{32,33}$ More specifically, local immune cells have been shown to secrete inflammatory cytokines and additional molecular mediators that function on the peripheral nerve terminals of nociceptor neurons. ${ }^{4}$ In response to inflammatory mediators, activated intracellular signaling pathways lead to a phosphorylation cascade, which reduces the threshold for nociceptor neurons to generate action potentials, eventually resulting in heightened pain sensitivity. ${ }^{34}$ Moreover, several inflammatory cytokines, such as TNF- $\alpha$, IL- $1 \beta$, IL- 6 , and IL-17, have been reported to directly change the responses of nociceptive neurons. ${ }^{35}$ Injection of TNF- $\alpha$, IL-6, and IL-17 into normal knees has been shown to lead to an increase in the C-fiber action potential frequency when knees were rotated in either a non-painful (less rotation) or painful (more rotation) manner. ${ }^{36-38}$ Likewise, IL-1 $\beta$ injection was also reported to increase the $\mathrm{C}$-fiber action potential frequency, but only 

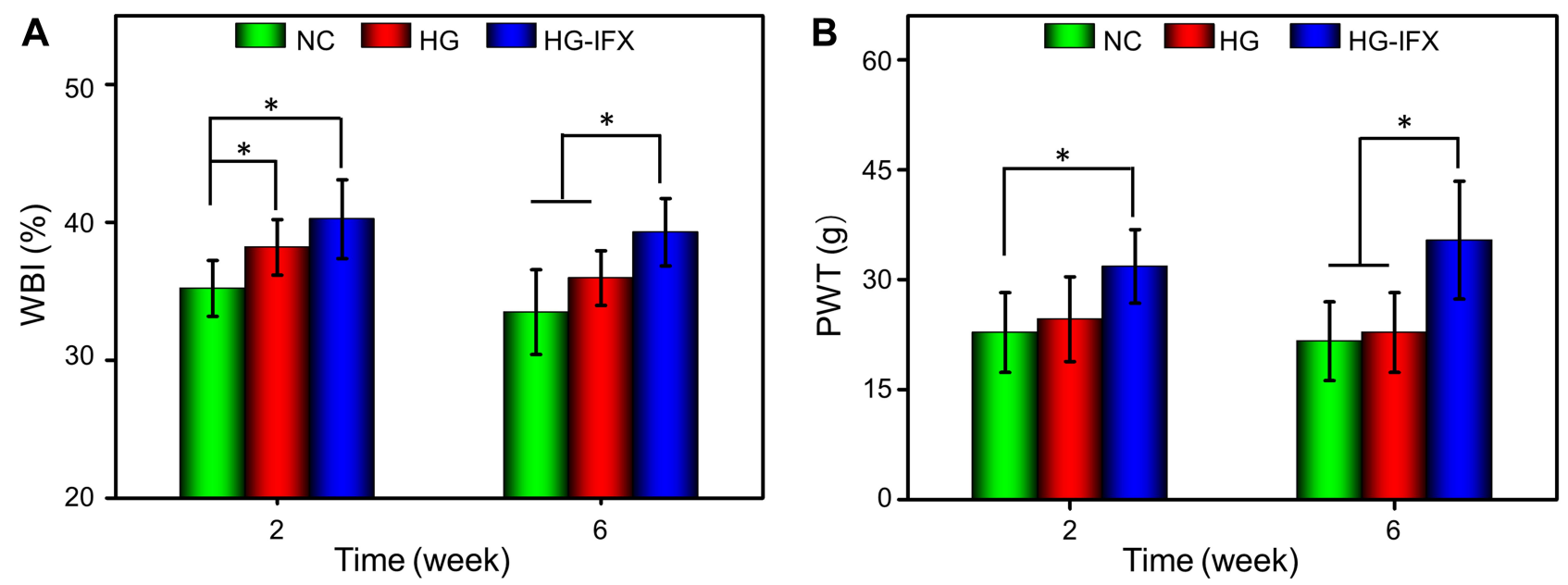

Figure 5 Changes of pain-related behavior after IFX-loaded hydrogel injection into the left knee joints. (A) Weight-bearing capacity was calculated by weight-bearing index (WBI). (B) Paw withdrawal threshold (PWT) was measured to test mechanical allodynia $\left(*_{\mathrm{p}}<0.05\right)$.

in response to painful rotation. ${ }^{39}$ Infliximab is a TNF inhibitor approved for the treatment of moderate-tosevere RA in the United States of America since $1999 .{ }^{40}$ In particular, IFX, which is a human-mouse chimeric monoclonal antibody, can rapidly bind to TNF- $\alpha$ with high affinity in both soluble and permeable forms, resulting in the generation of stable complexes and thus inhibiting the binding of TNF- $\alpha$ to receptors and depriving its biological activity. In addition, both in vitro and in vivo experiments have shown that in cells expressing the permeable form of TNF- $\alpha$, it could be dissolved following binding to IFX. ${ }^{40-42}$ Herein, administration of HG-IFX was shown to reduce inflammation by directly inhibiting TNF- $\alpha$, thereby downregulating the expression of a series of pain-related inflammatory cytokines (eg, IL-1 $\beta$, IL-6, and IL-17) and alleviating the pain symptoms of RA.

\section{Infliximab-Loaded Hydrogel Prevented Cartilage Destruction}

In patients with arthritis, articular cartilage destruction and disability caused by joint dysfunction are the most serious consequences. Therefore, the protection of the articular cartilage is essential for RA treatment. ${ }^{43}$ The macroscopic morphology of the articular surface would directly affect the extent of cartilage destruction. As shown in Figure 6A, images of the articular surface of the NC group revealed broad areas of cartilage damage with irregular surface defects at week 6 . Similar results were observed at the edge of the articular surface in the $\mathrm{HG}$ group. However, the cartilage in the HG-IFX group displayed a relatively intact surface, despite a slight surface fibrillation at the edge of the articular surface.

To further investigate the effects of IFX-loaded hydrogel on preventing cartilage damage, we performed $\mathrm{H} \& \mathrm{E}$, toluidine blue, and safranin $\mathrm{O}$-fast green staining, and immunohistochemical staining of COL I and COL II. Histological staining of the cartilage further confirmed the results of gross observation. As shown in Figure 6B, H\&E staining demonstrated that the articular surface was extremely unsmooth, even though some cartilages were missing and damaged, and the surface cells of the cartilage were necrotic (red allow) in the NC group. Regarding the cartilage in the HG group, the articular surface was not smooth, the surface cells of the cartilage had disappeared, and deep cells were vacuolated. In addition, cells were loosely and irregularly arranged, and suspected fibrous components could be observed on the surface of some cartilages (red allow). However, the articular cartilage in the HG-IFX group was shown to be generally intact, with cells being regularly arranged, but loosely and with few layers. Toluidine blue staining showed that the cartilage in the control group was seriously damaged, with the cartilage content being significantly decreased. However, the cartilage in the HG-IFX group was intact and abundant in content, whereas the cartilage in the $\mathrm{HG}$ group was demonstrated to be between that of the NC and HG-IFX groups (Figure 6C). In addition, we also performed safranin $\mathrm{O}$-fast green staining to analyze the cartilage matrix. We found that in the NC group, the cartilage was damaged, and glycoproteins were released in the cartilage, 

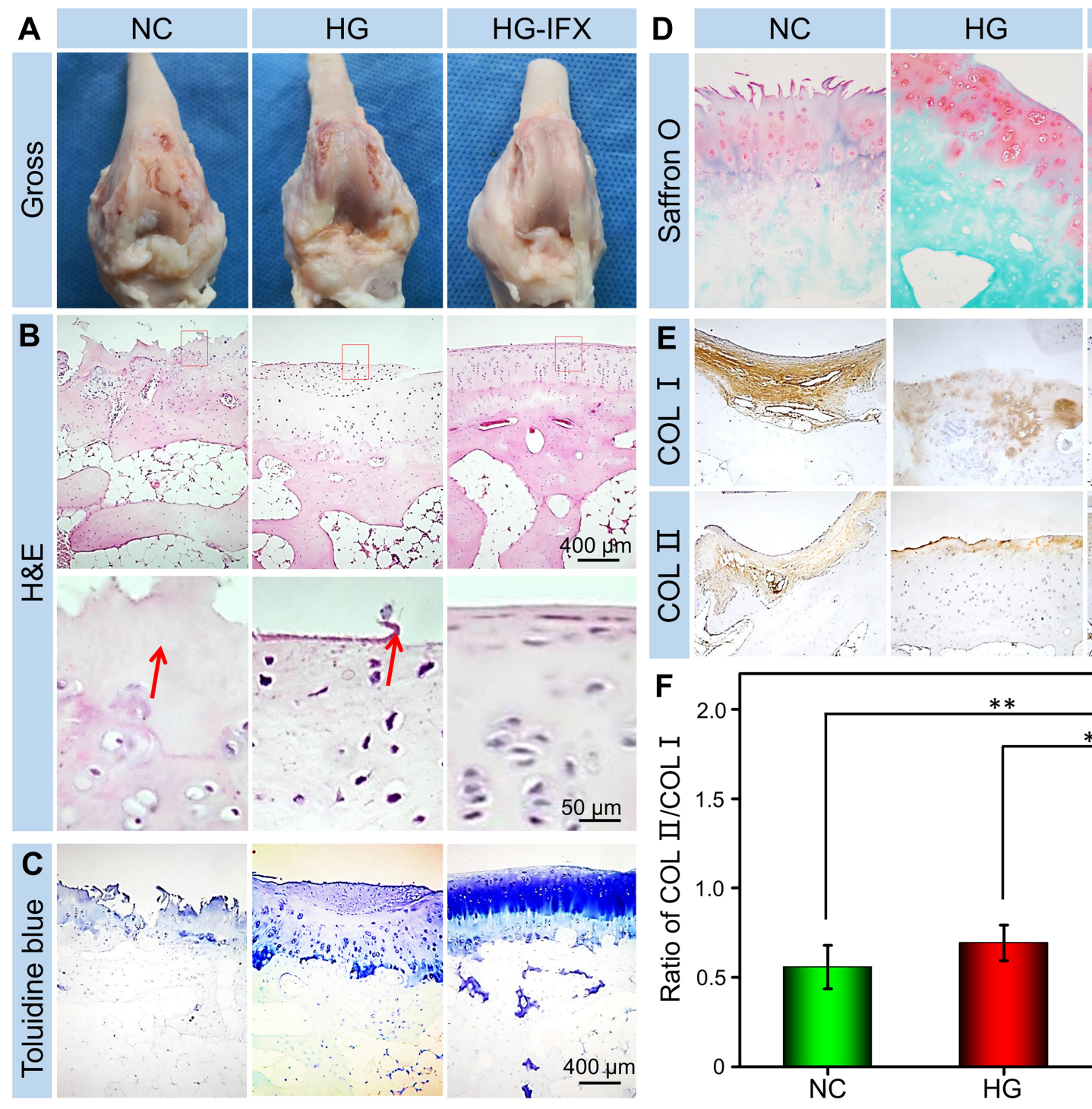

HG-IFX
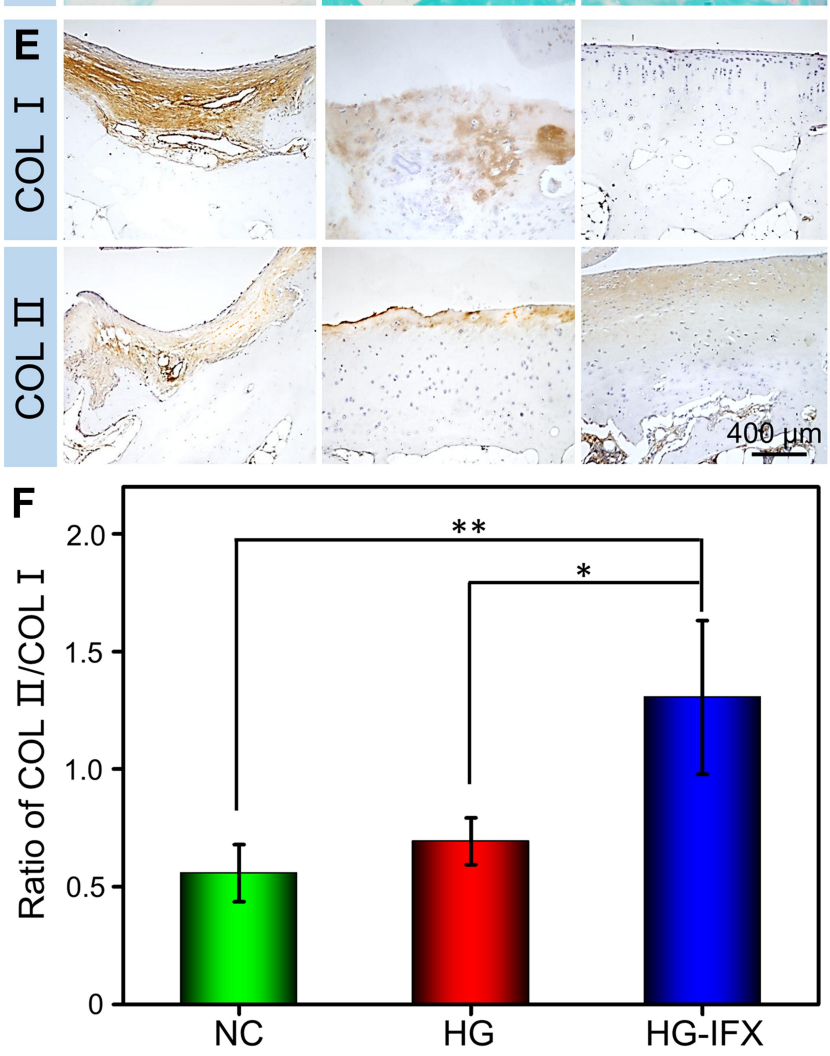

Figure 6 Macroscopic morphology and histological analyses of articular cartilage destruction. (A) Typical appearance of articular cartilage surface 6 weeks after implantation. (B) H\&E, (C) Toluidine blue, and (D) Safranin O-fast green staining of articular cartilage 6 weeks after implantation. Red arrows indicate the necrotic tissue on the surface of cartilage. (E) Immunohistochemical staining of COL I and COL II of articular cartilage 6 weeks after injection. (F) Ratio of COL II versus COL I calculated in the cartilage $\left({ }^{*} \mathrm{P}\right.$ $<0.05$, **p $<0.01$ ).

resulting in an uneven distribution of the matrix components and a slight or no coloration of safranin O. In the HG-IFX group, cartilage had a better morphology and matrix distribution (Figure 6D). In the case of immunohistochemical staining of COL I and COL II, we noted the limited expression of COL II, which is produced by chondrocytes, whereas COL I was detectable in reasonable quantities in the $\mathrm{NC}$ group (Figure 6E). In contrast, in the IFX-HG group, the expression of COL II in the cartilage was shown to be higher and well distributed, and the ratio of COL II versus COL I was higher in comparison with those in the NC and HG groups (Figure 6F). These results demonstrated that the content and quality of cartilage was inferior in the $\mathrm{NC}$ group and superior in the IFX-HG group.

\section{Improvement of Synovial Inflammation}

In RA, synovial inflammation and hyperplasia are the initiating factors, leading to invasive tissue mass finally causing joint destruction. Meanwhile, immune cells are known to secrete inflammatory cytokines and chemokines, which invade the cartilage and activate osteoclasts to cause bone degeneration. ${ }^{44}$ To investigate the severity of RA synovial inflammation and hyperplasia, we performed H\&E staining of synovial tissues around the left knee joints of RA rabbits (Figure 7). 


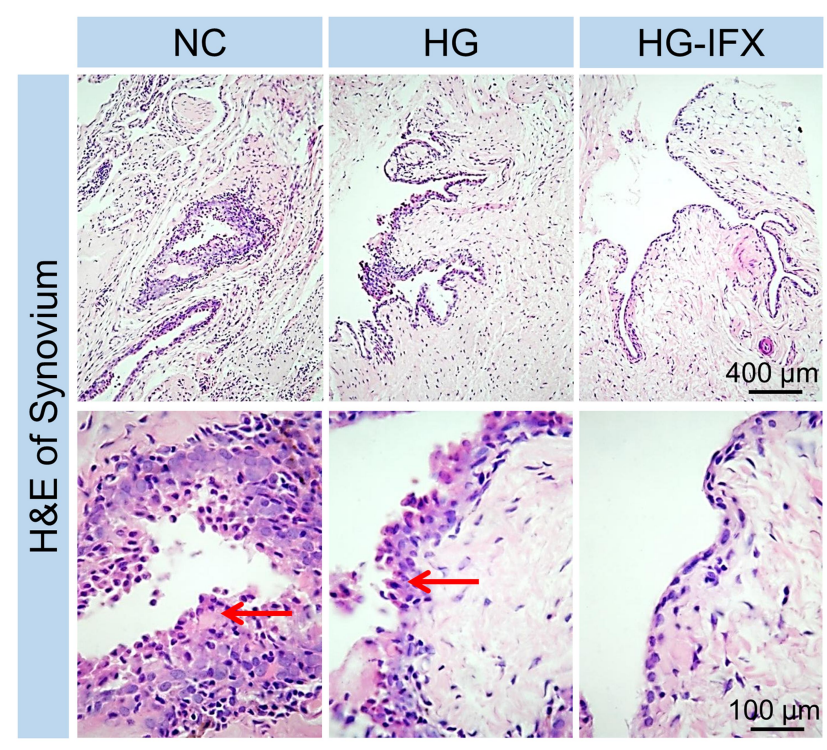

Figure 7 Improvement of synovial inflammation treated with IFX-loaded hydrogel. Typical synovium in each group stained with H\&E at 6 weeks post-injection (red arrows indicate synovial hyperplasia and inflammatory cell infiltration).

Accordingly, our results showed that the NC group had obvious synovial epithelial hyperplasia and inflammatory cell infiltration (red allow). These phenomena, however, were alleviated in the HG group. Compared with the NC and HG groups, the HG-IFX group was characterized by decreased inflammatory cell infiltration in the synovium, as well as mitigated hyperplasia of the synovial lining.

\section{Inhibition of the Expression of Inflammatory Cytokines in the Synovial Fluid}

We also detected levels of inflammatory cytokines in RA rabbits treated with saline, hydrogel, and IFXloaded hydrogel because these cytokines are known to drive RA progression, thus constituting another indicator of therapeutic efficacy. ${ }^{45}$ We noted that the levels of TNF- $\alpha$ in the synovial fluid were significantly inhibited when animals were treated with IFX-loaded hydrogel compared with animals treated either with $\mathrm{HG}$ or NC at both 2 and 6 week $(\mathrm{p}<0.001)$. Concomitantly, we observed a downregulation of the expression of IL-1 $\beta$, IL-6, and IL-17 in the synovial fluid in the IFX-HG group (Figure 8A and B). In addition, intraarticular injection with hydrogel alone was shown to also decrease the expression of some inflammatory factors, specifically inhibiting the expression of TNF- $\alpha(\mathrm{p}<0.05)$ and IL-1 $\beta(\mathrm{p}<$
$0.05)$ at 2 week and suppressing the expression of IL$1 \beta(p<0.05)$ at 6 week compared with those in the NC group.

\section{Inhibition of the Expression of Inflammatory Cytokines in Cartilage}

To further study the content of inflammatory factors in the cartilage, we performed immunofluorescence staining at 6 weeks post-injection. Similar to the results obtained for the levels of inflammatory cytokines in the synovial fluid, HG-IFX significantly inhibited the expression of TNF- $\alpha$ and IL-1 $\beta$ (Figure 9A), as well as IL-6 and IL-17 (Figure 9B) in the cartilage. In addition, using hydrogel alone also had a certain inhibitory effect on TNF- $\alpha$ and IL-6, compared with that in the NC group (Figure 9C).

Multiple immune-related cytokines, especially TNF$\alpha$, are known to play critical roles in the pathophysiology of autoimmune diseases, such as RA. TNF- $\alpha$ and IL-1 $\beta$ are pivotal factors that have been reported to result in a sustainable inflammatory reaction, cartilage destruction, and bone degeneration. ${ }^{46}$ Likewise, IL-6 is another important cytokine involved in the pathogenic mechanisms of RA. More specifically, IL-6 has been shown to induce differentiation of B-cells and Th17 cells, promoting the synthesis of acute phase proteins and matrix metalloproteinases (MMPs) to synergize with IL- $1 \beta{ }^{47}$ In RA, these inflammatory cytokines secreted by immune cells have been shown to result in synovial inflammation and hyperplasticity, and following invasion of the cartilage, lead to joint destruction. ${ }^{44}$ The joint protective results observed in this study might have been because IFX prevented the progression of local arthritis by inhibiting TNF- $\alpha$, thus downregulating the expression of numerous inflammatory cytokines (eg, IL-1 $\beta$, IL-6, and IL-17) and alleviating the erosion of cartilage induced by excessive inflammation.

TNF- $\alpha$, a critical cytokine related to RA, is known to play a crucial role in facilitating the proliferation of synovial cells and promoting the expression of other proinflammatory cytokines. ${ }^{48}$ TNF- $\alpha$ can induce the expression of inflammatory cytokines, such as IL-1 $\beta$ and IL-6, thus enhancing leukocyte migration. In addition, TNF- $\alpha$ can activate neutrophils and eosinophils and induce synovial cells or chondrocytes to produce tissuedegrading enzymes. ${ }^{49,50}$ Previous studies have shown 

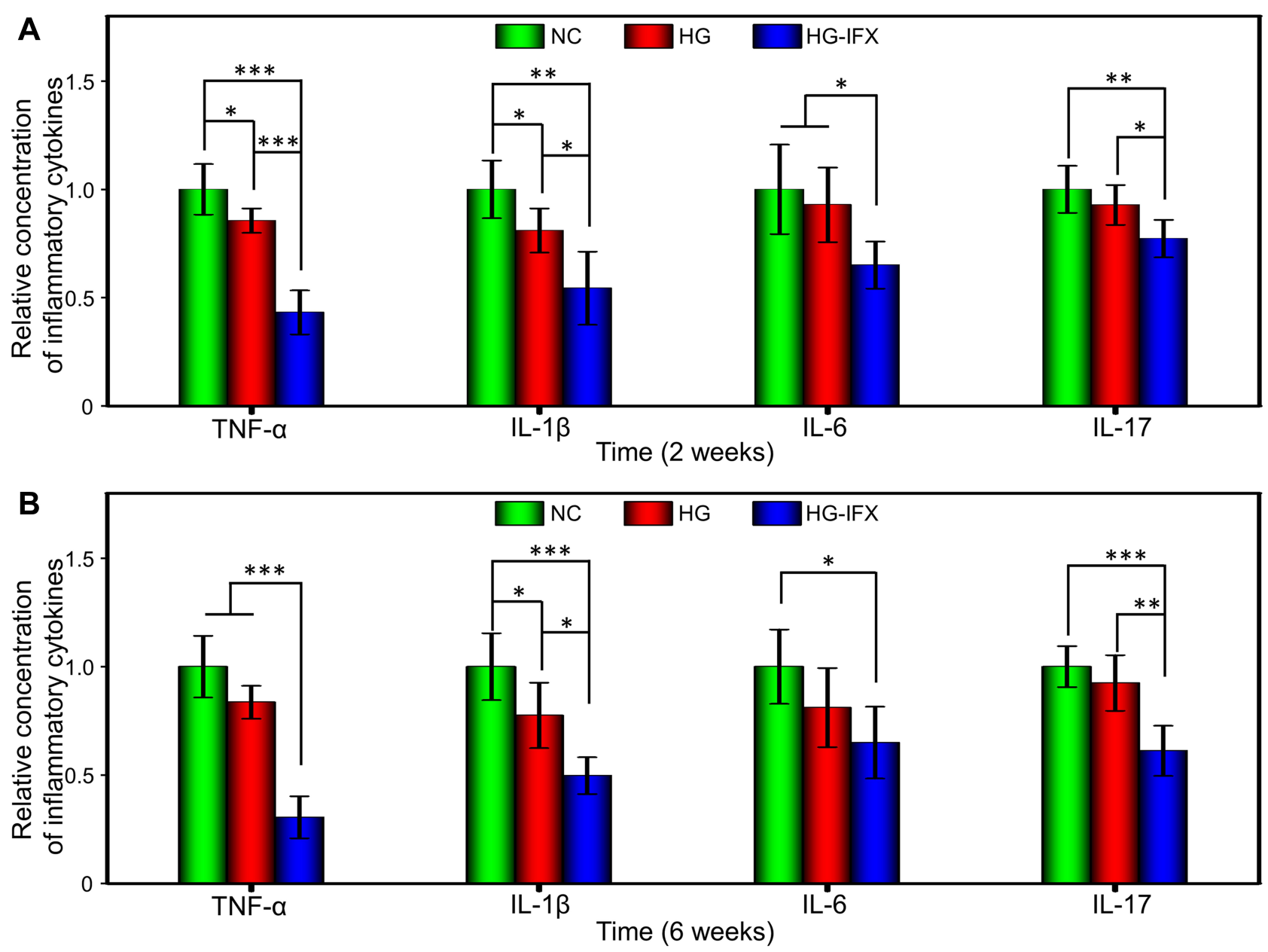

Figure 8 IFX-loaded hydrogel inhibited inflammatory cytokines expression in synovial fluid. The levels of inflammatory cytokines derived from synovial fluid of RA rabbits treated with saline, HG and HG-IFX at (A) 2 weeks and (B) 6 weeks post-injection $\left({ }^{*} \mathrm{p}<0.05, *^{*} \mathrm{p}<0.01, *^{*} \mathrm{p}<0.00 \mathrm{I}\right)$.

that excessive expression of TNF- $\alpha$ could induce cascade inflammatory reactions, thus causing pain and cartilage destruction, as well as could impede the chondrogenic ability of bone marrow stromal cells, further affecting the outcome of cartilage repair. ${ }^{51}$ In this study, intraarticular injection of a TNF- $\alpha$ inhibitor, IFX-loaded thermosensitive hydrogel employed as a local sustained-release system, significantly inhibited the expression of TNF- $\alpha$ in the synovial fluid and cartilage, thus downregulating the expression of other related inflammatory cytokines (eg, IL-1 $\beta$, IL-6, and IL-17). It further suppressed the progression of inflammation, thus alleviating pain and delaying cartilage destruction in RA. Due to the complex mechanisms of RA, further treatments for pain and cartilage destruction under arthritic conditions should be based on the development of modified scaffolds, application of proper marrow stromal cell sources, combination of pharmacotherapeutics, and addition of inducible factors. However, maintaining the structural and mechanical integrity of cartilage in RA remains a challenge. Regarding clinical application, we should note that this novel method to remedy RA still remains a rough attempt and highlight that it only provides a short-term therapeutic effect on early RA conditions. Moreover, further studies are needed to verify the underlying mechanisms.

\section{Conclusion}

In this study, a complex formulation, namely, an injection of an IFX-loaded thermosensitive and biocompatible hydrogel into the intraarticular space, was performed for treatment of an OVA-induced arthritis model in rabbits. Notably, intraarticular injection of this IFXencapsulating HA-based hydrogel could ameliorate the clinical symptoms of surface temperature and joint 

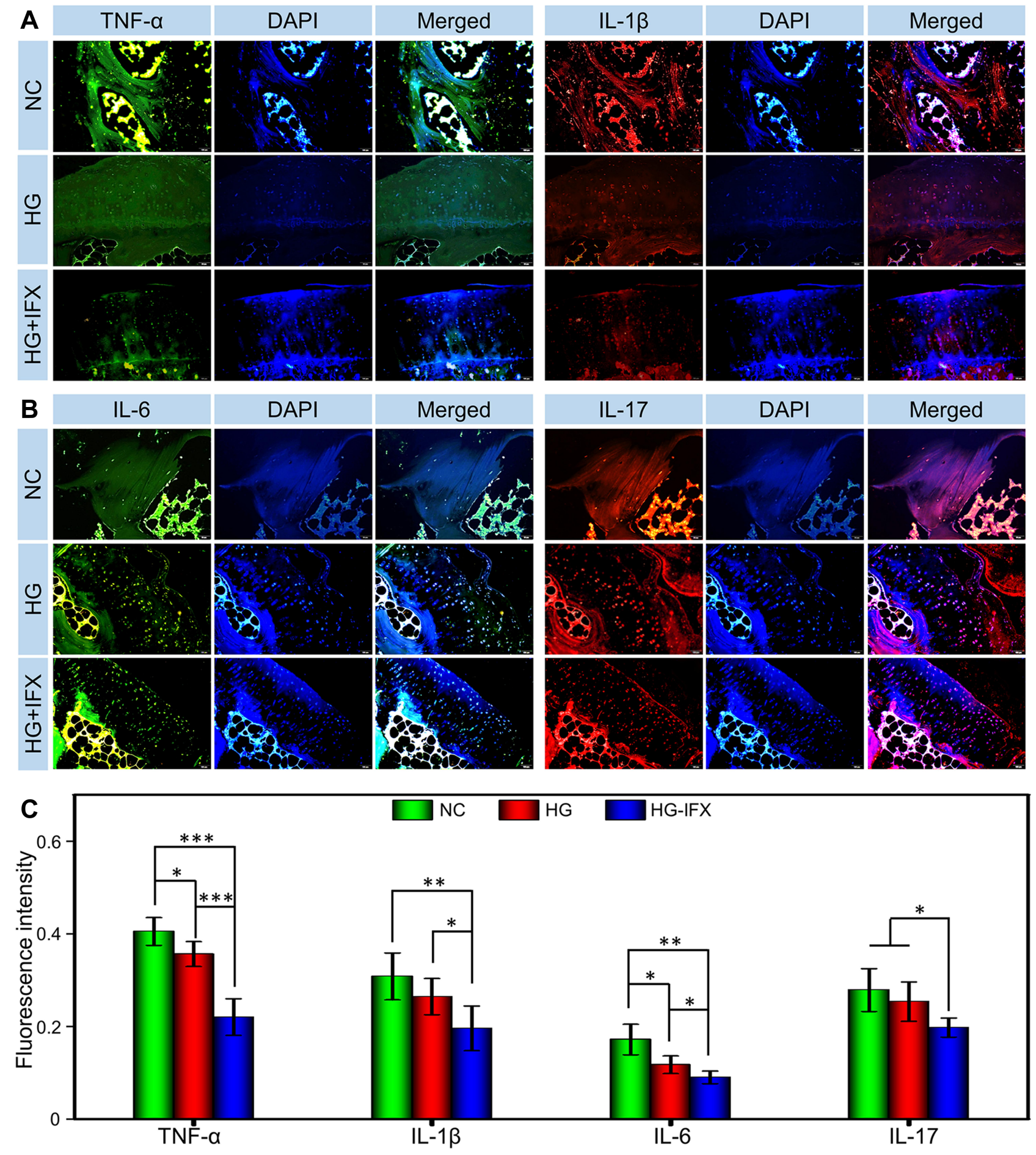

Figure 9 Inhibiting inflammatory cytokines expression in cartilage. Representative immunofluorescence images of (A) TNF- $\alpha$ and IL-I $\beta$, (B) IL-6 and IL-I7 expression in cartilage 6 weeks post-injection. (C) Quantitative statistics of TNF- $\alpha$, IL-I $\beta$, IL-6 and IL-I7 expression in cartilage 6 weeks post-injection $\left({ }^{*} \mathrm{p}<0.05\right.$, $* * \mathrm{p}<0.0 \mathrm{I}$, $* * * \mathrm{p}<$ $0.001)$.

swelling, resulting in decreased levels of inflammatory cytokines. Furthermore, local sustained release of the TNF- $\alpha$ inhibitor (IFX) relieved pain, controlled synovial inflammation, and prevented cartilage destruction.
Briefly, intraarticular injection of an IFX-loaded hydrogel as a drug delivery system presents great prospect in the remission of RA, and amelioration of RA-induced pain and cartilage injury. 


\section{Abbreviations}

DMEM, Dulbecco's modified Eagle medium; H\&E, hematoxylin and eosin; HA, hyaluronic acid; PBS, phosphate-buffered saline; PI, propidium iodide; PWT, paw withdrawal threshold; RA, rheumatoid arthritis; SD, Sprague-Dawley; SEM, scanning electron microscope; WBC, weight-bearing capacity; WBI, weight-bearing index.

\section{Data Sharing Statement}

The data that support the findings of this study are accessible from the corresponding author (Zhenzhou He) upon reasonable request.

\section{Author Contributions}

All authors made substantial contributions to conception and design, acquisition of data, or analysis and interpretation of data. All authors took part in drafting the article or in revising it; agreed to submit it to the current journal; gave final approval of the version to be published; and agree to be accountable for all aspects of the work.

\section{Funding}

This research was financially supported by Cultivation Program from the South Campus, Renji Hospital of Shanghai Jiaotong University for National Natural Science Foundation (Grant No. 2019GZRPYQN03).

\section{Disclosure}

The authors report no conflicts of interest in this work.

\section{References}

1. Taylor PC, Keystone EC, van der Heijde D, et al. Baricitinib versus placebo or adalimumab in rheumatoid arthritis. $N$ Engl $J$ Med. 2017;376(7):652-662. doi:10.1056/NEJMoa1608345

2. Yousri NA, Bayoumy K, Elhaq WG, et al. Large scale metabolic profiling identifies novel steroids linked to rheumatoid arthritis. Sci Rep. 2017;7(1):9137.

3. Vande Walle L, Van Opdenbosch N, Jacques P, et al. Negative regulation of the NLRP3 inflammasome by A20 protects against arthritis. Nature. 2014;512(7512):69-73. doi:10.1038/nature13322

4. Zhang A, Lee YC. Mechanisms for joint pain in rheumatoid arthritis (RA): from cytokines to central sensitization. Cur Osteoporosis Rep. 2018;16(5):603-610. doi:10.1007/s11914-018-0473-5

5. Krock E, Jurczak A, Svensson CI. Pain pathogenesis in rheumatoid arthritis-what have we learned from animal models? Pain. 2018;159 (Suppl 1):S98-S109.

6. Doan T, Massarotti E. Rheumatoid arthritis: an overview of new and emerging therapies. J Clin Pharmacol. 2005;45(7):751-762.

7. Fang G, Zhang Q, Pang Y, Thu HE, Hussain Z. Nanomedicines for improved targetability to inflamed synovium for treatment of rheumatoid arthritis: multi-functionalization as an emerging strategy to optimize therapeutic efficacy. $J$ Control Release. 2019;303:181-208.
8. Lu YC, Chuang $\mathrm{CH}$, Chuang $\mathrm{KH}$, et al. Specific activation of pro-Infliximab enhances selectivity and safety of rheumatoid arthritis therapy. PLoS Biol. 2019;17(6):e3000286.

9. Yang $\mathrm{X}, \mathrm{Du} \mathrm{H}$, Zhai G. Progress in intra-articular drug delivery systems for osteoarthritis. Curr Drug Targets. 2014;15(9):888-900.

10. Edwards SHR. Intra-articular drug delivery: the challenge to extend drug residence time within the joint. Veterinary J. 2011;190 (1):15-21. doi:10.1016/j.tvj1.2010.09.019

11. Brown S, Kumar S, Sharma B. Intra-articular targeting of nanomaterials for the treatment of osteoarthritis. Acta Biomater. 2019;93:239-257. doi:10.1016/j.actbio.2019.03.010

12. Mertz N, Ostergaard J, Yaghmur A, Larsen SW. Transport characteristics in a novel in vitro release model for testing the performance of intra-articular injectables. Int $J$ Pharm. 2019;566:445-453. doi:10.1016/j.ijpharm.2019.04.083

13. Badri W, Miladi K, Nazari QA, Greige-Gerges H, Fessi H, Elaissari A. Encapsulation of NSAIDs for inflammation management: overview, progress, challenges and prospects. Int J Pharm. 2016;515 (1-2):757-773. doi:10.1016/j.ijpharm.2016.11.002

14. Jung Y-S, Park W, Park H, Lee D-K, Na K. Thermo-sensitive injectable hydrogel based on the physical mixing of hyaluronic acid and Pluronic F-127 for sustained NSAID delivery. Carbohydr Polym. 2017;156:403-408. doi:10.1016/j.carbpol.2016.08.068

15. Li Y, Cao J, Han S, et al. ECM based injectable thermo-sensitive hydrogel on the recovery of injured cartilage induced by osteoarthritis. Artif Cells, Nanomed Biotechnol. 2018;46(sup2):152-160. doi:10.1080/21691401.2018.1452752

16. Kim T, Seol DR, Hahm S-C, et al. Analgesic effect of intra-articular injection of temperature-responsive hydrogel containing bupivacaine on osteoarthritic pain in rats. Biomed Res Int. 2015;2015:812949. doi:10.1155/2015/812949

17. Kim M, Hwang Y, Tae G. The enhanced anti-tissue adhesive effect of injectable pluronic-HA hydrogel by poly( $\gamma$-glutamic acid). Int J Biol Macromol. 2016;93(Pt B):1603-1611. doi:10.1016/j.ijbiomac.2016. 02.064

18. Oh SH, Kang JG, Lee JH. Co-micellized pluronic mixture with thermo-sensitivity and residence stability as an injectable tissue adhesion barrier hydrogel. J Biom Mater Res Part B. 2018;106 (1):172-182. doi:10.1002/jbm.b.33824

19. Seo J, Park SH, Kim MJ, et al. Injectable click-crosslinked hyaluronic acid depot to prolong therapeutic activity in articular joints affected by rheumatoid arthritis. ACS Appl Mater Interfaces. 2019;11(28):24984-24998. doi:10.1021/acsami.9b04979

20. Silva CR, Babo PS, Gulino M, et al. Injectable and tunable hyaluronic acid hydrogels releasing chemotactic and angiogenic growth factors for endodontic regeneration. Acta Biomater. 2018;77: 155-171. doi:10.1016/j.actbio.2018.07.035

21. Segal B, Rhodus NL, Patel K. Tumor necrosis factor (TNF) inhibitor therapy for rheumatoid arthritis. Oral Surgery. 2008;106(6):778-787. doi:10.1016/j.tripleo.2008.07.025

22. Fong Y, Chen C-H, Chen J-P. Intratumoral delivery of doxorubicin on folate-conjugated graphene oxide by in-situ forming thermo-sensitive hydrogel for breast cancer therapy. Nanomaterials. 2017;7(11):11. doi:10.3390/nano7110388

23. Micheli M, Bozdag B, Akgul A, et al. Pain relieving effect of-NSAIDs-CAIs hybrid molecules: systemic and intra-articular treatments against rheumatoid arthritis. Int J Mol Sci. 2019;20(8):8. doi:10.3390/ijms20081923

24. Tarafder S, Bose BS. Polycaprolactone-coated 3D printed tricalcium phosphate scaffolds for bone tissue engineering: in vitro alendronate release behavior and local delivery effect on in vivo osteogenesis. ACS Appl Mater Interfaces. 2014;6(13):9955-9965. doi:10.1021/am501048n

25. Posadowska U, Parizek M, Filova E, et al. Injectable nanoparticle-loaded hydrogel system for local delivery of sodium alendronate. Int J Pharmaceut. 2015;485(1-2):31-40. doi:10.1016/j. ijpharm.2015.03.003 
26. Aletaha D, Neogi T, Silman AJ, et al. 2010 Rheumatoid arthritis classification criteria: an American College of Rheumatology/ European League Against Rheumatism collaborative initiative. Arthritis Rheum. 2010;62(9):2569-2581. doi:10.1002/art.27584

27. Ayhan E. Intraarticular injections (corticosteroid, hyaluronic acid, platelet rich plasma) for the knee osteoarthritis. World J Orthop. 2014;5(3):351-361. doi:10.5312/wjo.v5.i3.351

28. Gouveia VM, Lopes-de-Araujo J, Costa Lima SA, Nunes C, Reis S. Hyaluronic acid-conjugated $\mathrm{pH}$-sensitive liposomes for targeted delivery of prednisolone on rheumatoid arthritis therapy. Nanomedicine. 2018;13(9):1037-1049. doi:10.2217/nnm-2017-0377

29. Yang H, Liu Z, Song Y, Hu C. Hyaluronic acid-functionalized bilosomes for targeted delivery of tripterine to inflamed area with enhancive therapy on arthritis. Drug Deliv. 2019;26(1):820-830. doi:10.1080/10717544.2019.1636423

30. Yu C, Li X, Hou Y, et al. Hyaluronic acid coated acid-sensitive nanoparticles for targeted therapy of adjuvant-induced arthritis in rats. Molecules. 2019;24:1.

31. Guidolin D, Franceschi F. Viscosupplementation with high molecular weight native hyaluronan. Focus on a 1500-2000 KDa fraction (Hyalubrix(R)). Eur Rev Med Pharmacol Sci. 2014;18(21):3326-3338.

32. Boyden SD, Hossain IN, Wohlfahrt A, Lee YC. Non-inflammatory causes of pain in patients with rheumatoid arthritis. Curr Rheumatol Rep. 2016;18(6):30. doi:10.1007/s11926-016-0581-0

33. Meeus M, Vervisch S, De Clerck LS, Moorkens G, Hans G, Nijs J. Central sensitization in patients with rheumatoid arthritis: a systematic literature review. Semin Arthritis Rheum. 2012;41 (4):556-567. doi:10.1016/j.semarthrit.2011.08.001

34. Pinho-Ribeiro FA, Verri WA, Chiu IM. Nociceptor sensory neuronimmune interactions in pain and inflammation. Trends Immunol. 2017;38(1):5-19. doi:10.1016/j.it.2016.10.001

35. Schaible H-G. Nociceptive neurons detect cytokines in arthritis. Arthritis Res Ther. 2014;16(5):470. doi:10.1186/s13075-014-0470-8

36. Richter F, Natura G, Loser S, Schmidt K, Viisanen H, Schaible H-G. Tumor necrosis factor causes persistent sensitization of joint nociceptors to mechanical stimuli in rats. Arthritis Rheum. 2010;62 (12):3806-3814. doi:10.1002/art.27715

37. Brenn D, Richter F, Schaible H-G. Sensitization of unmyelinated sensory fibers of the joint nerve to mechanical stimuli by interleukin-6 in the rat: an inflammatory mechanism of joint pain. Arthritis Rheum. 2007;56(1):351-359. doi:10.1002/art.22282

38. Richter F, Natura G, Ebbinghaus M, et al. Interleukin-17 sensitizes joint nociceptors to mechanical stimuli and contributes to arthritic pain through neuronal interleukin-17 receptors in rodents. Arthritis Rheum. 2012;64(12):4125-4134. doi:10.1002/art.37695

39. Ebbinghaus M, Uhlig B, Richter F, et al. The role of interleukin-1 $\beta$ in arthritic pain: main involvement in thermal, but not mechanical, hyperalgesia in rat antigen-induced arthritis. Arthritis Rheum. 2012;64(12):3897-3907. doi:10.1002/art.34675
40. Zrubka Z, Gulacsi L, Brodszky V, et al. Long-term efficacy and cost-effectiveness of infliximab as first-line treatment in rheumatoid arthritis: systematic review and meta-analysis. Expert Rev Pharmacoecon Outcomes Res. 2019;19(5):537-549. doi:10.1080/ 14737167.2019.1647104

41. Atzeni F, Sarzi-Puttini P, Botsios C, et al. Long-term anti-TNF therapy and the risk of serious infections in a cohort of patients with rheumatoid arthritis: comparison of adalimumab, etanercept and infliximab in the GISEA registry. Autoimmun Rev. 2011;190 (1):225-229. doi:10.1016/j.autrev.2012.06.008

42. Isgren A, Forslind K, Erlandsson M, et al. High survivin levels predict poor clinical response to infliximab treatment in patients with rheumatoid arthritis. Semin Arthritis Rheum. 2018;106 (1):652-657. doi:10.1016/j.semarthrit.2011.08.005

43. Zhao Y, Wang Z, Jiang Y, et al. Biomimetic composite scaffolds to manipulate stem cells for aiding rheumatoid arthritis management. Adv Funct Mater. 2019;1807860.

44. Kasama T, Isozaki T, Takahashi R, Miwa Y. Clinical effects of tocilizumab on cytokines and immunological factors in patients with rheumatoid arthritis. Int Immunopharmacol. 2016;35:301-306.

45. Wang P, Tao JH, Pan HF. Probiotic bacteria: a viable adjuvant therapy for relieving symptoms of rheumatoid arthritis. Inflammopharmacology. 2016;24(5):189-196.

46. Ansboro S, Roelofs AJ, De Bari C. Mesenchymal stem cells for the management of rheumatoid arthritis: immune modulation, repair or both? Curr Opin Rheumatol. 2017;29(2):201-207.

47. Li C, Li H, Wang Q, et al. pH-sensitive polymeric micelles for targeted delivery to inflamed joints. J Control Release. 2017; 246:133-141.

48. Kotake S, Nanke Y. Effect of TNFalpha on osteoblastogenesis from mesenchymal stem cells. Biochim Biophys Acta. 2014;1840 (3):1209-1213.

49. Su CM, Hu SL, Sun Y, et al. Myostatin induces tumor necrosis factor-alpha expression in rheumatoid arthritis synovial fibroblasts through the PI3K-Akt signaling pathway. J Cell Physiol. 2019;234 (6):9793-9801.

50. Goodman SM, Menon I, Christos PJ, Smethurst R, Bykerk VP. Management of perioperative tumour necrosis factor alpha inhibitors in rheumatoid arthritis patients undergoing arthroplasty: a systematic review and meta-analysis. Rheumatology. 2016;55 (3):573-582.

51. Papadaki HA, Kritikos HD, Gemetzi C, et al. Bone marrow progenitor cell reserve and function and stromal cell function are defective in rheumatoid arthritis: evidence for a tumor necrosis factor alpha-mediated effect. Blood. 2002;99(5):1610-1619.
Journal of Pain Research

\section{Publish your work in this journal}

The Journal of Pain Research is an international, peer reviewed, open access, online journal that welcomes laboratory and clinical findings in the fields of pain research and the prevention and management of pain. Original research, reviews, symposium reports, hypothesis formation and commentaries are all considered for publication. The manuscript

Submit your manuscript here: https://www.dovepress.com/journal-of-pain-research-journa management system is completely online and includes a very quick and fair peer-review system, which is all easy to use. Visit http:// www.dovepress.com/testimonials.php to read real quotes from published authors. 